\title{
Article \\ Strong Dipole-Quadrupole-Exciton Coupling Realized in a Gold Nanorod Dimer Placed on a Two-Dimensional Material
}

\author{
Huajian Pang ${ }^{1,+}$, Hongxin Huang ${ }^{1,+}$, Lidan Zhou ${ }^{1}$, Yuheng Mao ${ }^{1}$, Fu Deng ${ }^{2, *}$ and Sheng Lan ${ }^{1, *(\mathbb{D})}$ \\ 1 Guangdong Provincial Key Laboratory of Nanophotonic Functional Materials and Devices, \\ School of Information and Optoelectronic Science and Engineering, South China Normal University, \\ Guangzhou 510006, China; hj.pang@foxmail.com (H.P.); hongxin_huang@foxmail.com (H.H.); \\ zhould@mail2.sysu.edu.cn (L.Z.); erik_maoyh@m.scnu.edu.cn (Y.M.) \\ 2 Department of Physics, The Hong Kong University of Science and Technology, Kowloon, Hong Kong, China \\ * Correspondence: fu_deng@foxmail.com (F.D.); slan@scnu.edu.cn (S.L.) \\ + The authors contributed equally to this work.
}

Citation: Pang, H.; Huang, H.;

Zhou, L.; Mao, Y.; Deng, F.; Lan, S.

Strong Dipole-Quadrupole-Exciton

Coupling Realized in a Gold Nanorod Dimer Placed on a Two-Dimensional

Material. Nanomaterials 2021, 11, 1619.

https://doi.org/10.3390/

nano11061619

Academic Editor: Kosei Ueno

Received: 30 May 2021

Accepted: 18 June 2021

Published: 20 June 2021

Publisher's Note: MDPI stays neutral with regard to jurisdictional claims in published maps and institutional affiliations.

Copyright: (c) 2021 by the authors. Licensee MDPI, Basel, Switzerland. This article is an open access article distributed under the terms and conditions of the Creative Commons Attribution (CC BY) license (https:// creativecommons.org/licenses/by/ $4.0 /)$.

\begin{abstract}
Simple systems in which strong coupling of different excitations can be easily realized are highly important, not only for fundamental research but also for practical applications. Here, we proposed a T-shaped gold nanorod (GNR) dimer composed of a long GNR and a short GNR perpendicular to each other and revealed that the dark quadrupole mode of the long GNR can be activated by utilizing the dipole mode excited in the short GNR. It was found that the strong coupling between the dipole and quadrupole modes can be achieved by exciting the T-shaped GNR dimer with a plane wave. Then, we demonstrated the realization of strong dipole-quadrupole-exciton coupling by placing a T-shaped GNR on a tungsten disulfide $\left(\mathrm{WS}_{2}\right)$ monolayer, which leads to a Rabi splitting as large as $299 \mathrm{meV}$. It was confirmed that the simulation results can be well fitted by using a Hamiltonian based on the coupled harmonic oscillator model and the coupling strengths for dipole-quadrupole, dipole-exciton and quadrupole-exciton can be extracted from the fitting results. Our findings open new horizons for realizing strong plasmon-exciton coupling in simple systems and pave the way for constructing novel plasmonic devices for practical applications.
\end{abstract}

Keywords: two-dimensional material; scattering; strong coupling; Rabi splitting; quadrupole

\section{Introduction}

In recent years, systems in which light is strongly coupled with matter have attracted great interest because they not only act as platforms for investigating cavity quantum electrodynamics and quantum entanglement [1-4] but also exhibit potential applications in quantum operations [5], ultrafast optical switch [6], and low threshold lasers [7]. In a photon-exciton system, strong coupling occurs when the energy exchange rate between photons and excitons is fast enough to overcome the respective energy dissipation rates. In this case, two mixed states with the characteristics of both light and matter will be generated, which are generally referred to as polaritons. This behavior is manifested in the spectrum of the system as Rabi splitting $[1,4,8-10]$. If the system is in the weak coupling regime, no polariton state will be generated and the spontaneous decay rate is governed by the so-called Purcell effect [11].

It is well known that metallic nanoparticles support localized surface plasmon resonances (LSPRs) which provide sub-wavelength confinement of light on the surfaces of nanoparticles, leading to extremely small mode volumes. In addition, the significantly enhanced electric field and the tunable resonant wavelength offered by LSPRs render them ideal platforms for realizing strong plasmon-exciton coupling. In general, the strong coupling can be revealed in the scattering spectrum of a metallic nanoparticle as an anticrossing behavior with a large Rabi splitting exceeds the average damping rates of plasmons and excitons. Unlike bright plasmon modes, special excitation schemes are generally required for dark plasmon modes [12-15]. For example, linearly polarized light was used 
to excite the anapole mode of slotted silicon nanodisks and the dipole mode of gold nanorods [16,17]. Meanwhile, strong coupling between the anapole mode or dipole mode and the dark mode was observed, exhibiting a large Rabi splitting.

Recent studies have demonstrated the advantages of monolayer transition metal dichalcogenide (TMDC) in realizing strong photon-exciton or plasmon-exciton coupling because of their direct bandgaps [18], large exciton transition dipole moment [19], and extremely large absorption $\left(\mathrm{MoS}_{2}\right.$ and $\mathrm{WS}_{2}$ can reach $10 \%$ and $15 \%$, respectively at resonance) $[20,21]$. By designing resonant photonic structures, the absorption efficiency of TMDC can be enhanced [22]. More importantly, the binding energy of excitons in monolayer TMDCs is very large [23], implying that excitons can survive at room temperature. Thus far, the strong coupling between a TMDC monolayer and a photonic or plasmonic resonator has been successfully demonstrated, including optical microcavities [24,25], periodic nanostructures [26,27], single plasmonic nanoparticle antennas [28-34], and nanoparticleon-film systems [35-40].

For a strongly coupled system consisting of a single nanoparticle and a TMDC monolayer, the typical Rabi splitting energy ranges from 80 to $120 \mathrm{meV}$ [28-31]. Recently, strong coupling between excitons and the anapole mode formed by the interference of Mie resonances, which leads to a Rabi splitting of $\sim 190 \mathrm{meV}$, has been observed in nanodisks made of multilayer $\mathrm{WS}_{2}$ [41]. In order to explore the rich physics of polaritons, however, it is highly desirable to further enhance the coupling strength between plasmons and excitons. On the one hand, it was predicted that the nonradiative dark mode can enhance the coupling strength between a nanocavity and quantum emitters [42]. On the other hand, the strong coupling of three excitations has become the focus of many studies. By additionally introducing a microcavity, plasmons, excitons and photons can be mixed to create three polaritonic states. When the system enters into the strong coupling regime, Rabi splitting larger than 300 or even $500 \mathrm{meV}$ can be realized [25,43]. In addition, it was demonstrated that strong exciton-plasmon-polariton coupling with an effective energy separation exceeding $410 \mathrm{meV}$ can be achieved by fabricating a $\mathrm{WS}_{2}$ grating on a gold ( $\mathrm{Au}$ ) film [44]. However, it remains a challenge to realize strong plasmon-exciton coupling with an enhanced coupling strength in a single plasmonic nanostructure on a TMDC monolayer, which can be simply characterized by measuring the scattering spectrum of the nanostructure. Very recently, strong anapole-plasmon-exciton coupling is realized by introducing a plasmonic antenna into a $\mathrm{WSe}_{2}$-anapole hybrid system, leading to an enhanced Rabi splitting of $\sim 159 \mathrm{meV}$ [45]. Unfortunately, complex nanofabrication techniques are necessary for realizing strong coupling in such a hybrid system.

In this article, we proposed the use of a hybrid system composed of a T-shaped GNR dimer placed on a $\mathrm{WS}_{2}$ monolayer to realize strong dipole-quadrupole-exciton coupling. We showed that the dark quadrupole mode in a long GNR can be excited by using the dipole mode of a short GNR placed nearby, forming a T-shaped GNR dimer. The dipole mode excited in the short GNR couples strongly with the quadrupole mode in the long GNR, resulting a Rabi splitting of $\sim 255 \mathrm{meV}$. We demonstrated that strong dipolequadrupole-exciton coupling can be achieved when the T-shaped dimer is placed on a $\mathrm{WS}_{2}$ monolayer, leading to a Rabi splitting as large as $\sim 299 \mathrm{meV}$. The eigen-energies of the mixed states and the coupling strengths for dipole-quadrupole, dipole-exciton and quadrupoleexciton were extracted from the fitting of the numerical simulation results with the coupled oscillator model in which two and three oscillators are involved. The strong coupling is confirmed not only by an anti-crossing behavior revealed in the two-dimensional scattering and absorption spectra but also by the criterion of strong coupling.

\section{Materials and Methods}

In this work, a commercial software developed by Lumerical Solution Inc. (https://www.lumerical.com, accessed on 30 May 2021) was used for the numerical simulation based on three-dimensional finite-difference time-domain (FDTD) technique. The dielectric function of Au was taken from the experimental data [46], while that of $\mathrm{WS}_{2}$ 
was taken from previous literature [20]. The exciton energy in $\mathrm{WS}_{2}$ monolayer was chosen to be $2.016 \mathrm{eV}$. The diameter of the GNRs was chosen to be $40 \mathrm{~nm}$ and the thickness of the $\mathrm{WS}_{2}$ monolayer was set to be $1.0 \mathrm{~nm}$. For simplicity, we considered mainly the systems suspended in air and discussed the effects of a silica $\left(\mathrm{SiO}_{2}\right)$ substrate on the simulation results from the viewpoint of practical implementation. In the numerical simulations, we used non-uniform grids with the smallest size of $0.5 \mathrm{~nm}$ in all directions. In addition, a perfectly matched layer boundary condition was employed to absorb all outgoing waves.

\section{Results and Discussion}

We first investigated the excitation of the quadrupole mode supported by a GNR. Since it cannot be directly excited by using a plane wave, it is considered as a dark mode. However, it has been shown that a dipole source can be used as one of the effective ways to excite this mode [12], as schematically shown in Figure 1a. The diameter of the GNR was fixed to be $D=40 \mathrm{~nm}$. The dipole source was placed $40 \mathrm{~nm}$ away from the center of the GNR and the dipole moment was made perpendicular to the long axis of the GNR.
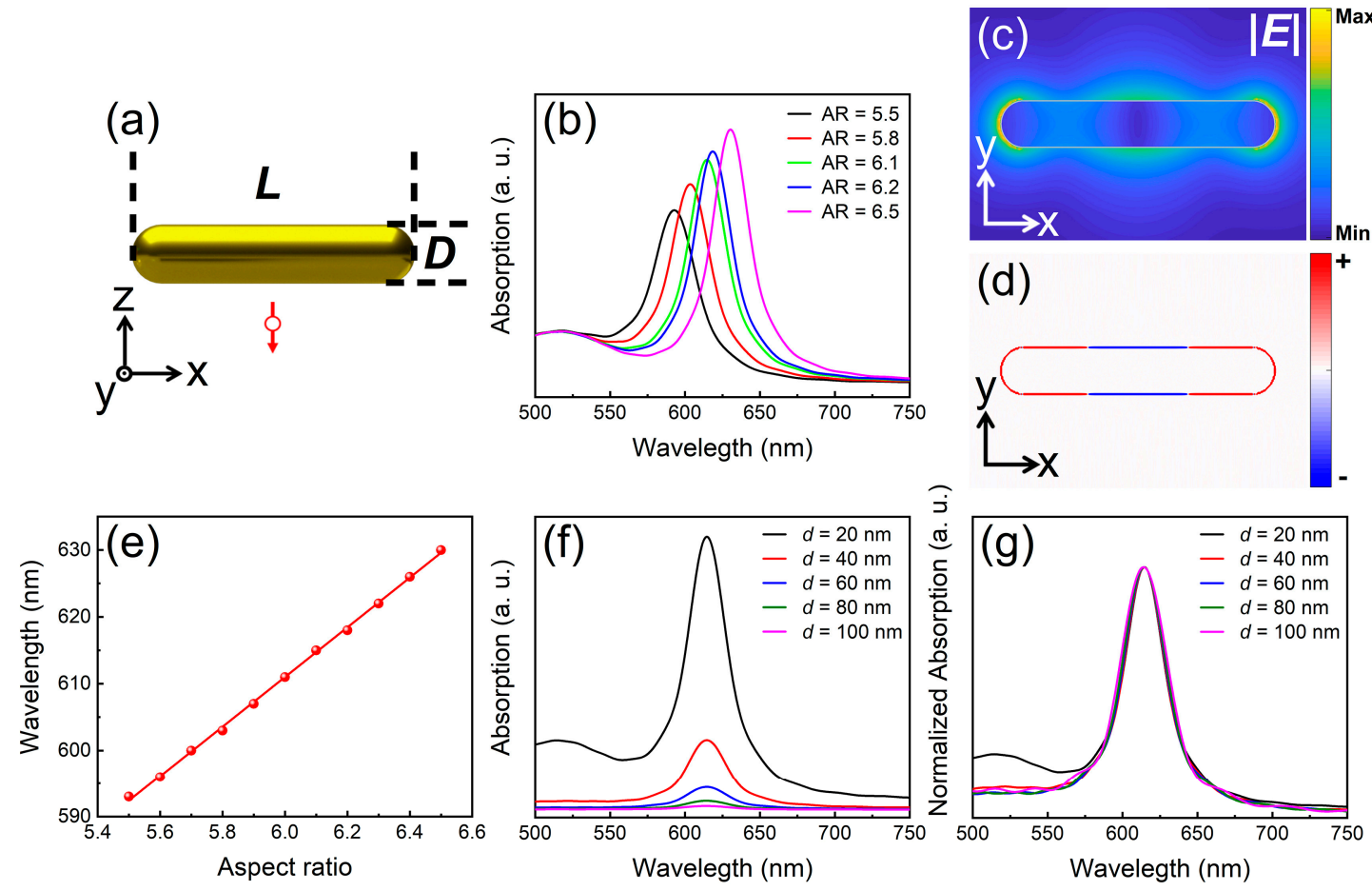

Figure 1. Excitation of the quadrupole mode in a GNR by using a dipole source. (a) Schematic showing a GNR with a length of $L$ and a diameter $D$ and a dipole source placed nearby. (b) Absorption spectra calculated for GNRs with different aspect ratios. Electric field (c) and surface charge (d) distributions calculated for a GNR with AR $=6.1$ at $\lambda=615 \mathrm{~nm}$. (e) Dependence of the resonance wavelength on the aspect ratio of the GNR. (f) Absorption spectra calculated for the long GNR (AR $=6.1$ ), which is excited by a dipole source placed at different distances $(d)$. $(\mathrm{g})$ Normalized absorption spectra of the long GNR shown in (f).

In Figure 1b, we show the absorption spectra calculated for GNRs with aspect ratios (AR), which is defined as $\mathrm{AR}=L / D$, ranging from 5.5 to 6.5. A redshift of the absorption peak as well as a narrowing of the linewidth are observed when the AR of the GNR is increased, as shown in Figure 1b. In order to verify that the absorption of the GNR arises from the excitation of the quadrupole mode, we calculated the electric field and surface charge distributions of a GNR with $\mathrm{AR}=6.1$ at the absorption peak $(\sim 615 \mathrm{~nm})$, as shown in Figure 1c,d, respectively. Actually, the GNR acts as an F-P cavity which supports high-order plasmon modes with electric field distributions similar to standing waves along the long axis of the GNR [47]. The N-order plasmon mode is identified as N nodes in the electric field distribution. In Figure 1c, one can find two nodes in the electric field distribution, 
implying the excitation of the quadrupole mode. This assignment is further confirmed by the surface charge distribution shown in Figure 1d, which exhibits two electric dipole moments oscillating oppositely. In this case, the electric dipole of the GNR disappears completely. In Figure 1e, we present the dependence of the resonant wavelength of the quadrupole mode on the AR of the GNR, which shows a linear relationship. It means that one can readily tune the resonant wavelength of the quadrupole mode by simply varying the aspect ratio of the GNR. Then, we investigated the effects of the distance between the dipole source and the GNR surface $(d)$ on the excitation of the quadrupole mode, as shown in Figure 1f,g. It was noticed that the absorption of the GNR decreases rapidly with increasing the distance. When we inspected the normalized absorption spectra, which are presented in Figure $1 \mathrm{~g}$, it was found that the resonant wavelength (i.e., the absorption peak) and the linewidth remained unchanged. Therefore, we conclude that the distance between the GNR and the dipole source only influences the excitation efficiency of the quadrupole mode of the GNR.

As demonstrated above, the quadrupole mode of a GNR can be excited by a dipole source placed nearby. We replaced the dipole source with another GNR with a much shorter length, forming a T-shaped GNR dimer, as shown in Figure 2a. The short GNR supports only electric dipole mode which can be easily activated by using a plane wave incident from the top. In Figure 2b, we present the scattering spectra calculated for GNR dimers with different gap widths $(w)$. In this case, the lengths of the long and short GNRs are chosen to be $L_{1}=248 \mathrm{~nm}$ and $L_{\mathrm{S}}=84 \mathrm{~nm}$, respectively. The scattering spectrum of the isolated short GNR and the absorption spectrum of the isolated long GNR are also shown for comparison. It is noticed that the scattering spectrum of the GNR dimer, which is dominated by a single peak for a large gap width $(w=60 \mathrm{~nm})$, evolves gradually into two peaks with identical intensities at a small gap width $(w=10 \mathrm{~nm})$. This behavior indicates that the dipole mode of the short GNR, which is activated by the incident plane wave, excites successfully the dark quadrupole mode of the long GNR. In addition, these two modes coupled with each other, resulting in two mixed plasmon modes. The coupling strength between the two modes, which is manifested in the energy separation between the two scattering peaks, increases with decreasing gap width. The intensities of the two scattering peaks become equal when the gap width is reduced to be $w=10 \mathrm{~nm}$, as shown in Figure $2 \mathrm{~b}$. As the GNR dimer is excited by using a plane wave polarized along the long axis of the short GNR, the transverse localized plasmon resonance of the long GNR, which is a dipole mode, will also be activated. It appears as a small protrusion at $\sim 500 \mathrm{~nm}$ in the scattering spectrum of the GNR dimer. In Figure 2c, we show the two-dimensional scattering spectra calculated for GNR dimers composed of short GNRs with different lengths. The gap width is fixed at $w=10 \mathrm{~nm}$. An anti-crossing behavior is clearly observed, verifying the coupling between the dipole mode of the short GNR and the quadrupole mode of the long GNR. The Hamiltonian based coupled harmonic oscillator model can be used to fit the anti-crossing behavior. It can be expressed as [48]:

$$
\hat{H}=h\left(\begin{array}{cc}
E_{\mathrm{D}}-i \frac{\gamma_{\mathrm{D}}}{2} & g_{\mathrm{D}-\mathrm{Q}} \\
g_{\mathrm{D}-\mathrm{Q}} & E_{\mathrm{Q}}-i \frac{\gamma_{\mathrm{Q}}}{2}
\end{array}\right)
$$

Here, $E_{\mathrm{D}}$ and $E_{\mathrm{Q}}$ represent the energies of uncoupled dipole and quadrupole resonances, $\gamma_{\mathrm{D}}$ and $\gamma_{\mathrm{Q}}$ denote the dissipation rates of the uncoupled dipole and quadrupole modes, respectively, and $g_{\mathrm{D}-\mathrm{Q}}$ stands for the dipole-quadrupole coupling strength. The Hopfield coefficient in each mixed state, which represents the contributions of the dipole and quadrupole modes in the mixed state, can be extracted from the Hamiltonian of the coupled system [49]. 
(a)

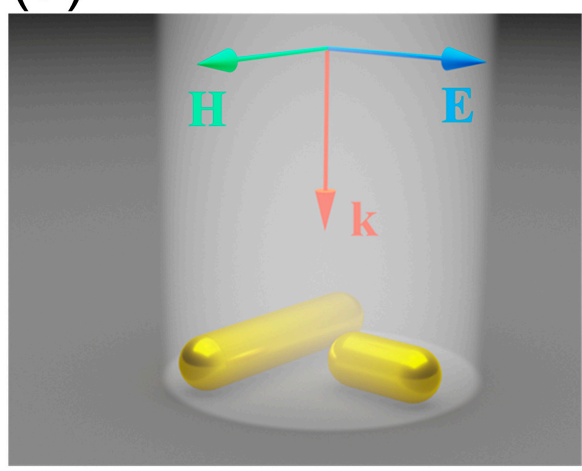

(c)

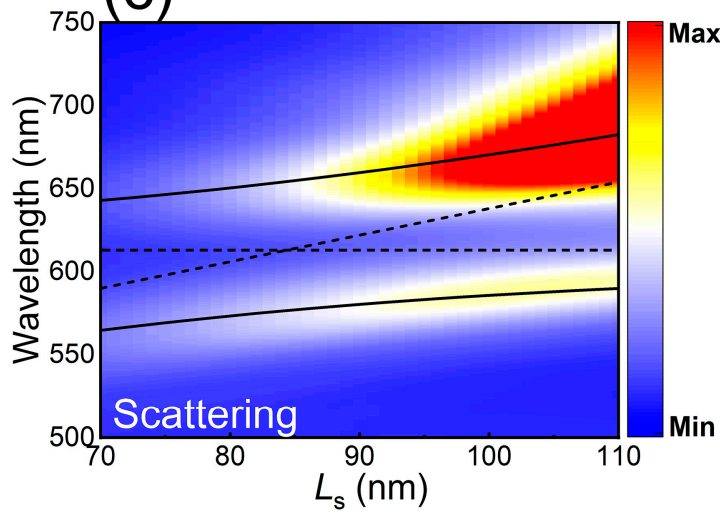

(b)

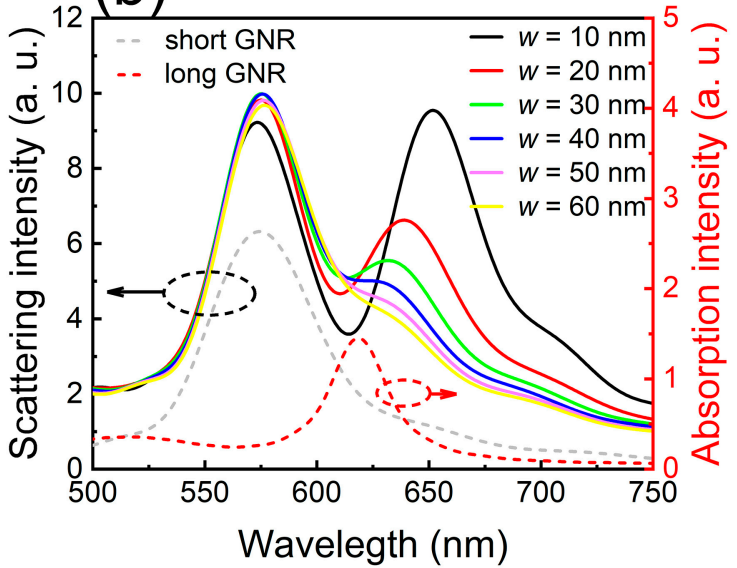

(d)

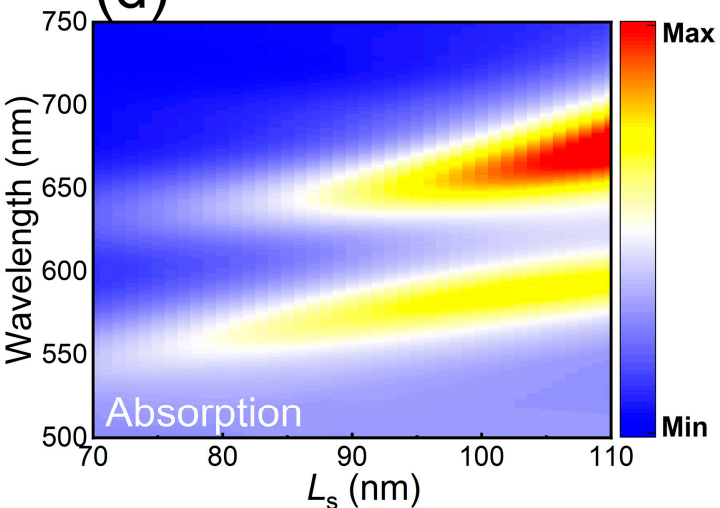

Figure 2. Strong coupling between the dipole and quadrupole modes excited in a T-shaped GNR dimer. (a) Schematic showing the T-shaped GNR dimer composed of a long and a short GNR and the wavevector and the electric/magnetic field of the plane wave used to excite the GNR dimer. (b) Scattering spectra calculated for GNR dimers with different gap widths $(w)$. The scattering spectrum of the isolated short GNR (gray dashed curve) and the absorption spectrum of the isolated long GNR (red dashed curve) are also provided. (c) Two-dimensional scattering spectra calculated for GNR dimers composed of short GNRs with different lengths. The fitting results based on Equation (1) are also provided. (d) Two-dimensional absorption spectra calculated for GNR dimers composed of short GNRs with different lengths.

The two branches formed by dipole-quadrupole coupling can be fitted by using Equation (1), as shown in Figure 2c. The solid curves represent the high-energy $\left(E_{\mathrm{H}}\right)$ and low-energy $\left(E_{\mathrm{L}}\right)$ branches of the mixed states while the dotted lines represent the uncoupled dipole $\left(E_{\mathrm{D}}\right)$ and quadrupole $\left(E_{\mathrm{Q}}\right)$ modes. The Rabi splitting is derived to be $\Omega \sim 255 \mathrm{meV}$ when $E_{\mathrm{D}}=E_{\mathrm{Q}}$. The linewidths of the $E_{\mathrm{D}}$ and $E_{\mathrm{Q}}$ modes used in the fitting are $\gamma_{\mathrm{D}} \approx 150 \mathrm{meV}$ and $\gamma_{\mathrm{Q}} \approx 110 \mathrm{meV}$, similar to the values reported previously $[47,50]$. It is found that the criterion for strong coupling is satisfied in this case, i.e., $\Omega>\left(\gamma_{\mathrm{D}}+\gamma_{\mathrm{Q}}\right) / 2$ [51], implying that the coupling between the dipole and quadrupole modes enters into the strong coupling regime. As shown in Figure $2 \mathrm{~d}$, the anti-crossing behavior revealed in the scattering spectra is also observed in the two-dimensional absorption spectra of the GNR dimers, further confirming the strong coupling of the dipole and quadrupole modes.

In order to gain a deep insight into the strong coupling behavior, we calculated the electric field and surface charge distributions of the GNR dimer at the scattering peaks $(\lambda=573$ and $652 \mathrm{~nm})$ and the scattering valley $(\lambda=615 \mathrm{~nm})$, as shown in Figure 3 . It was found that the electric field is localized in the gap region and the strongest one was observed at the low-energy peak $(\lambda=652 \mathrm{~nm})$. The excitation of the dipole mode is also evidenced in the electric field distribution of the short GNR. The dipole mode excited in the short GNR is more clearly reflected in the surface charge distribution, as shown in 
Figure $3 \mathrm{~d}-\mathrm{f}$. At the high-energy peak $(\lambda=573 \mathrm{~nm})$, one can see the interaction between the two dipoles excited in the short and long GNRs. In contrast, a dipole-quadrupole interaction is identified at the low-energy peak $(\lambda=652 \mathrm{~nm})$. It is noticed that charges with opposite signs are excited on the two sides of the gap region, implying the establishment of a strong electric field (see Figure 3c), which is crucial for realizing strong plasmon-exciton coupling described in the following.

(a)

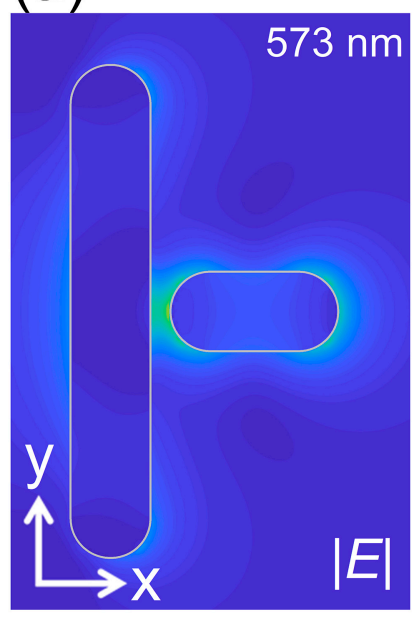

(d)

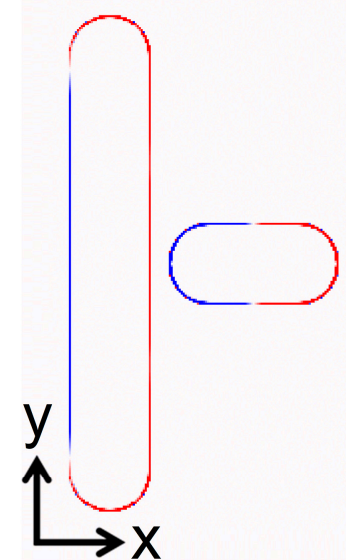

(b)

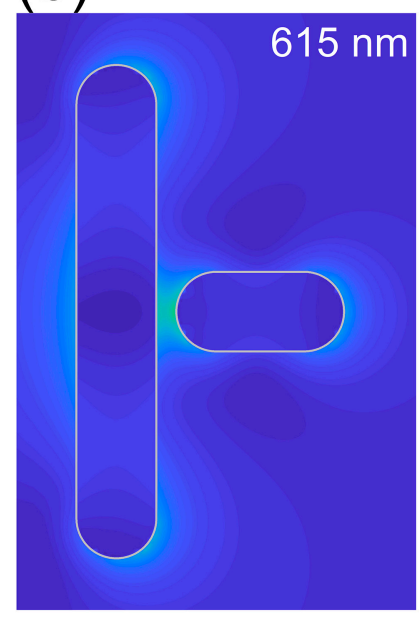

(e)

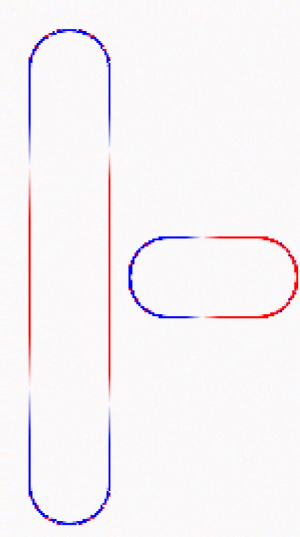

(c)

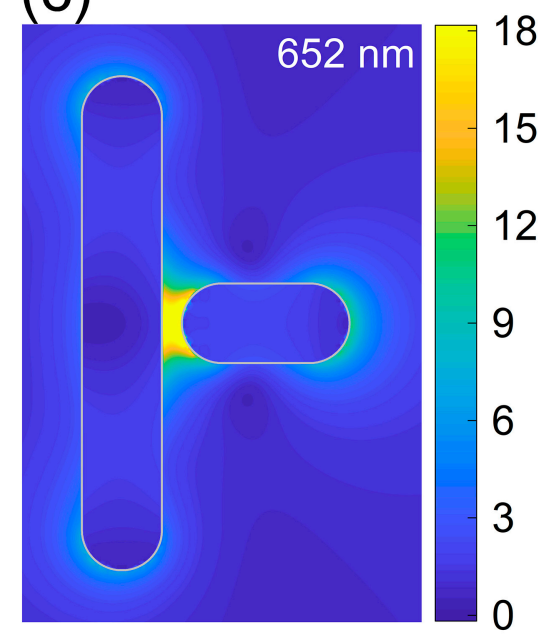

(f)

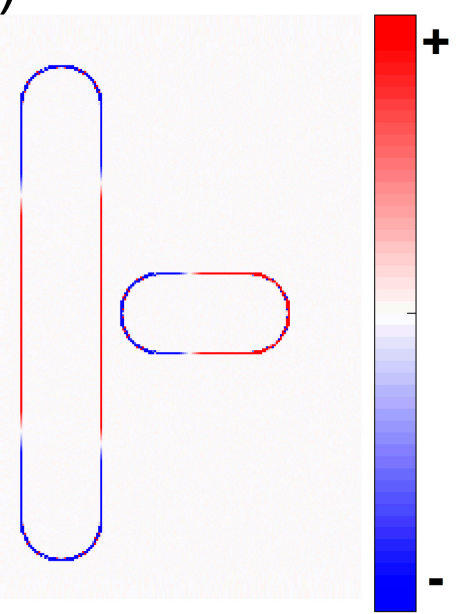

Figure 3. Electric field (upper panel) and surface charge (lower panel) distributions calculated for the T-shaped GNR with $L_{1}=248 \mathrm{~nm}, L_{\mathrm{s}}=84 \mathrm{~nm}$ and $w=10 \mathrm{~nm}$ at different wavelengths. $(\mathbf{a}, \mathbf{d}) \lambda=573 \mathrm{~nm},(\mathbf{b}, \mathbf{e}) \lambda=615 \mathrm{~nm},(\mathbf{c}, \mathbf{f}) \lambda=652 \mathrm{~nm}$.

Then, we examined the coupling between the dipole mode of a GNR and the excitons in a $W_{2}$ monolayer. In this case, the GNR was placed on top of the monolayer $\mathrm{WS}_{2}$ and a plane wave whose polarization was along the axis of the GNR was incident normally on the GNR, as schematically illustrated in Figure $4 \mathrm{a}$. For a GNR with $L_{\mathrm{s}}=94 \mathrm{~nm}$, a strong coupling between the dipole mode excited in the GNR and the excitons in the $\mathrm{WS}_{2}$ monolayer was achieved. As a result, the single peak in the scattering spectrum of the GNR was split into two peaks, corresponding to the two mixed states, as shown in Figure $4 \mathrm{~b}$. The red solid curve represents the absorption spectrum of the excitons in the $\mathrm{WS}_{2}$ monolayer used in the numerical simulation. It possesses a peak wavelength at $\sim 615 \mathrm{~nm}$ and a linewidth of $\sim 33 \mathrm{meV}$, consistent with the experimental data reported previously $[20,30,33]$. The scattering spectrum of the GNR in the absence of the $\mathrm{WS}_{2}$ monolayer, which is represented by the gray dotted curve, is also provided for comparison. If we plot the two-dimensional scattering spectra calculated for GNRs with different 
lengths, an anti-crossing behavior is clearly observed, as shown in Figure 4c. Similarly, the $E_{\mathrm{L}}$ and $E_{\mathrm{H}}$ branches of the plexcitons can be well fitted by using the Hamiltonian based the coupled harmonic oscillator model, which is written as:

$$
\hat{H}=h\left(\begin{array}{cc}
E_{\mathrm{D}}-i \frac{\gamma_{\mathrm{D}}}{2} & g_{\mathrm{D}-\mathrm{ex}} \\
g_{\mathrm{D}-\mathrm{ex}} & E_{\mathrm{ex}}-i \frac{\gamma_{\mathrm{ex}}}{2}
\end{array}\right)
$$

Here, $E_{\mathrm{D}}$ and $E_{\mathrm{ex}}$ represent the energies of uncoupled dipole and exciton resonances, $\gamma_{\mathrm{D}}$ and $\gamma_{\mathrm{ex}}$ denote the dissipation rates of the uncoupled dipole mode and exciton, respectively, and $g_{\mathrm{D}-\mathrm{ex}}$ stands for the dipole-exciton coupling strengths.

(a)

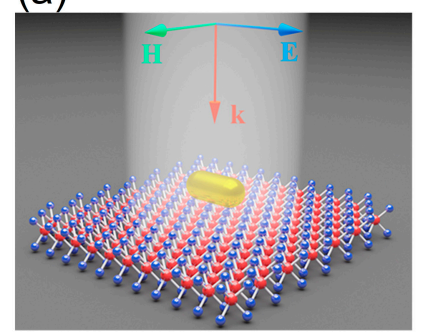

(d)
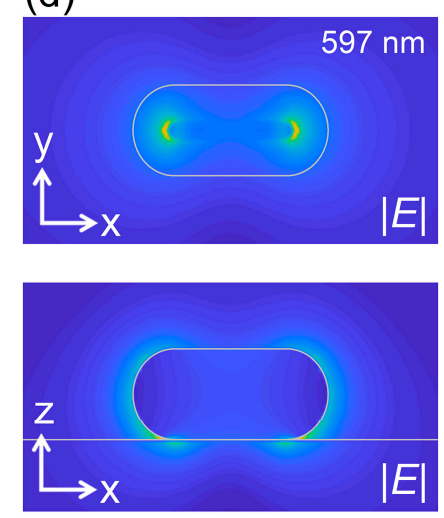

(b)

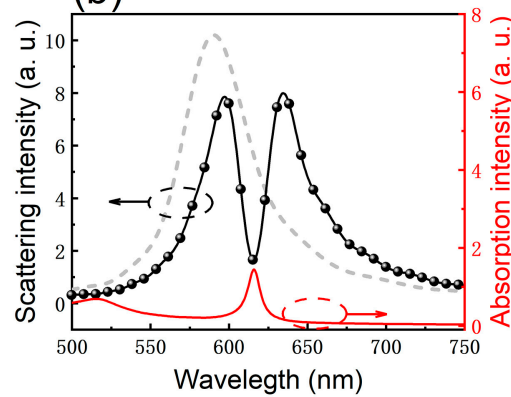

(e)
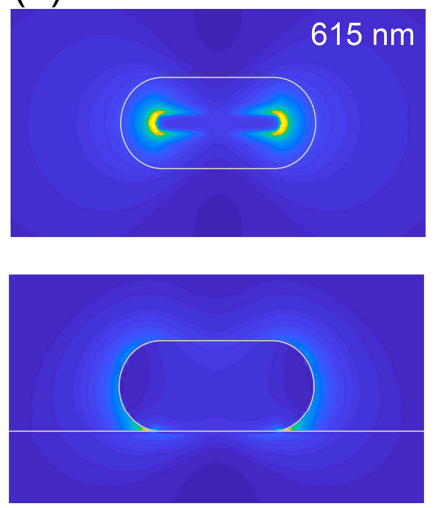

(c)

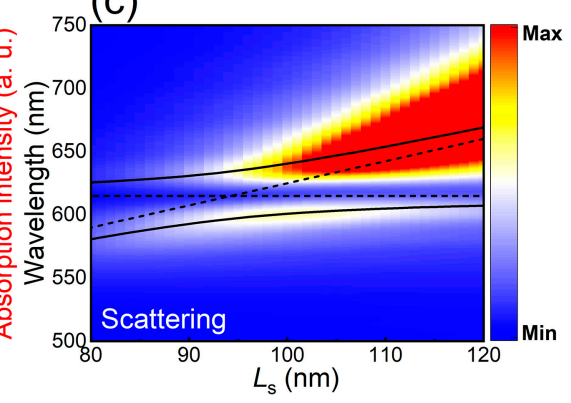

(f)

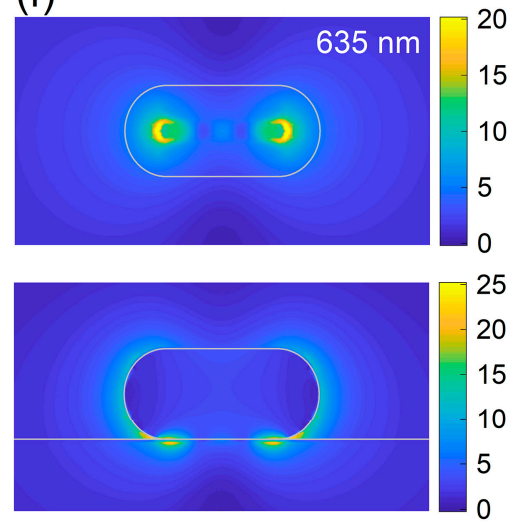

Figure 4. Strong coupling between the dipole mode in a single GNR and the excitons in a monolayer $\mathrm{WS}_{2}$. (a) $\mathrm{Schematic}$ showing the excitation of a single GNR placed on a monolayer $\mathrm{WS}_{2}$ by using plane wave. (b) Scattering spectrum (black solid curve) calculated for a GNR resonant with the exciton resonance of the monolayer $\mathrm{WS}_{2}$. Additionally, shown are the scattering spectrum of the GNR in the absence of the monolayer $\mathrm{WS}_{2}$ (dashed curve) and the absorption spectrum of the monolayer $\mathrm{WS}_{2}$ (red solid curve). (c) Two-dimensional scattering spectra calculated for GNRs with different lengths. The fitting results based on Equation (2) are also provided. (d-f) Electric field distributions in the XY plane (upper panel) and XZ plane (lower panel) calculated for the GNR with $L_{\mathrm{s}}=94 \mathrm{~nm}$ at different wavelengths of $\lambda=597,615$ and $635 \mathrm{~nm}$, respectively.

The two dashed lines interacting with each other represent the energies of the dipole mode and the exciton resonance in the absence of coupling. The Rabi splitting energy extracted from the fitting is $\Omega \sim 123 \mathrm{meV}$, which is basically consistent with the results of previous works [28]. This value is larger than the average damping rates of the dipole mode and the exciton resonance, implying that the plasmon-exciton coupling in this system enters into the strong coupling regime. Figure $4 \mathrm{~d}-\mathrm{f}$ shows the electric field distributions in the $X Y$ and XZ planes at the two scattering peaks $(\lambda=597$ and $635 \mathrm{~nm})$ and the scattering valley $(\lambda=615 \mathrm{~nm})$. It can be seen that the electric field is mainly localized on the contact surface with larger enhancements at the two ends of the GNR, indicating a strong interaction between the plasmons and the excitons.

After discussing the dipole-quadrupole coupling and dipole-exciton coupling, we studied the dipole-quadrupole-exciton coupling in a T-shaped GNR dimer placed on a $\mathrm{WS}_{2}$ monolayer, as schematically illustrated in Figure 5a. In this case, the length of the 
short GNR was chosen to be $L_{\mathrm{s}}=81 \mathrm{~nm}$ and the gap width was set to be $w=10 \mathrm{~nm}$. As shown in Figure 5b, one can identify three peaks in the scattering spectrum of the GNR dimer placed on a $W_{2}$ monolayer. They correspond to the three mixed states arising from the strong coupling among the dipole, quadrupole and exciton modes. The intensities of the two scattering peaks are equal and they are much stronger than that of the middle one. The scattering valleys at $\lambda=615 \mathrm{~nm}$ and $\lambda=645 \mathrm{~nm}$ correspond to the exciton resonance and the quadrupole mode, respectively. The resonant wavelength of the quadrupole mode is red shifted owing to the existence of the $\mathrm{WS}_{2}$ monolayer which possesses a large refractive index. With increasing $L_{\mathrm{s}}$, the dipole mode is red shifted, passing through the exciton and quadrupole modes successively. One can see two anti-crossings in the two-dimensional scattering spectra plotted for GNR dimers with different $L_{\mathrm{S}}$, as shown in Figure 5c. Based on the coupled harmonic oscillator model, the Hamiltonian used to fit the three mixed states of the hybrid system can be expressed as follows [25,30,43]:

$$
\hat{H}=h\left(\begin{array}{ccc}
E_{\mathrm{D}}-i \frac{\gamma_{\mathrm{D}}}{2} & g_{\mathrm{D}-\mathrm{Q}} & g_{\mathrm{D}-\mathrm{ex}} \\
g_{\mathrm{D}-\mathrm{Q}} & E_{\mathrm{Q}}-i \frac{\gamma_{\mathrm{Q}}}{2} & g_{\mathrm{Q}-\mathrm{ex}} \\
g_{\mathrm{D}-\mathrm{ex}} & g_{\mathrm{Q}-\mathrm{ex}} & E_{\mathrm{ex}}-i \frac{\gamma_{\mathrm{ex}}}{2}
\end{array}\right)
$$

(a)

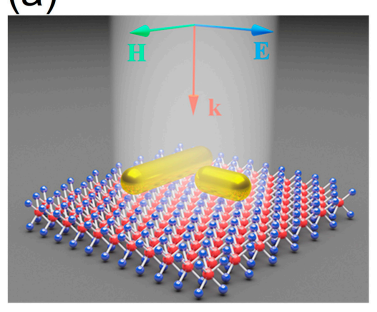

(c)

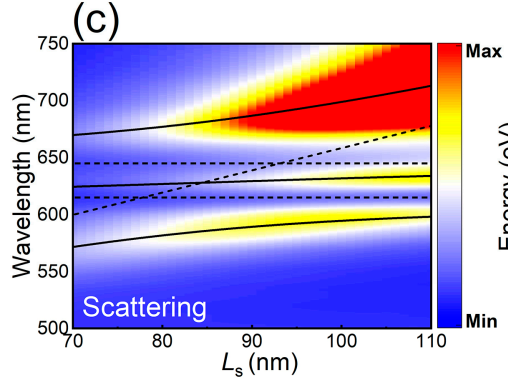

(b)

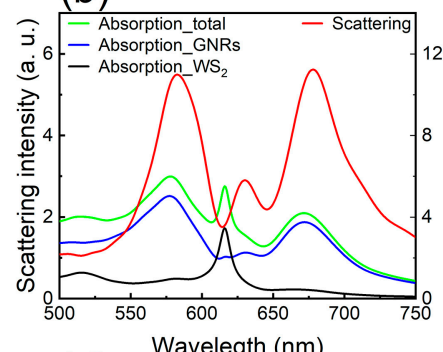

(d)

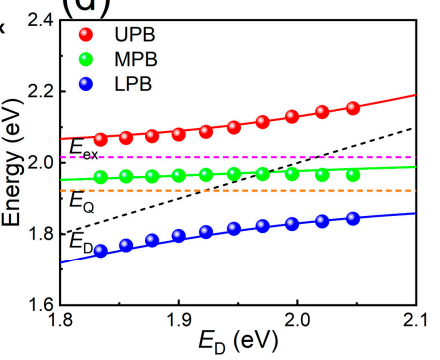

(e)

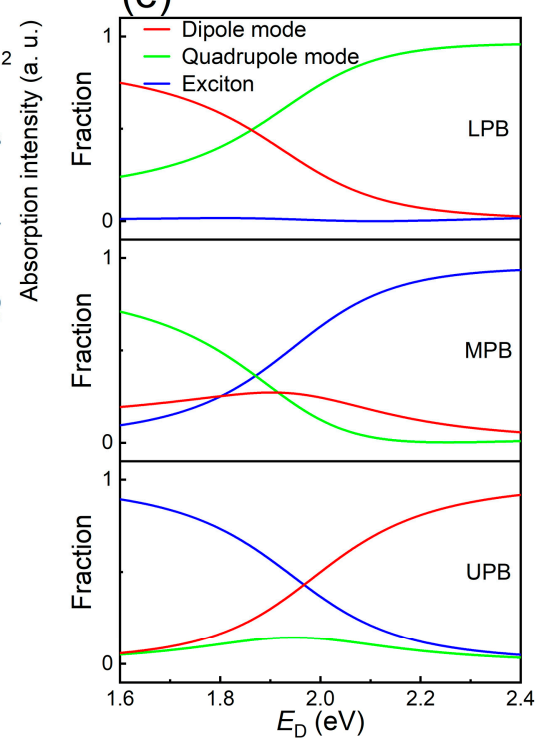

Figure 5. Strong dipole-quadrupole-exciton coupling realized in a T-shaped GNR dimer placed on a monolayer WS 2 . (a) Schematic showing a T-shaped GNR dimer placed on a monolayer $\mathrm{WS}_{2}$ and excited by a plane wave. (b) Scattering spectrum calculated for the GNR dimer placed on a $W S_{2}$ monolayer with $L_{1}=248 \mathrm{~nm}, L_{\mathrm{s}}=81 \mathrm{~nm}$ and $w=10 \mathrm{~nm}$. The absorption spectra of the long and short GNRs only and the absorption spectrum of the monolayer $\mathrm{WS}_{2}$ are also provided. (c) Two-dimensional scattering spectra calculated for GNR dimers composed of short GNRs with different lengths. (d) The red, green and blue circles represent the upper, middle and lower polariton branch (UPB, MPB and LPB) resonance energies extracted from the scattering spectrum of T-shaped GNR dimer placed on a $\mathrm{WS}_{2}$ monolayer hybrid structure. The red, green and blue solid lines represent the resonance energies of UPB, MPB and LPB fitted by Hamiltonian analysis Equation (3). The black, orange and pink dashed lines represent dipole, quadrupole and exciton resonances, respectively. (e) Hopfield coefficients for dipole, quadrupole and exciton modes contributions to UPB, MPB and LPB as a function of dipole resonance.

Here, $E_{\mathrm{D}}, E_{\mathrm{Q}}$ and $E_{\mathrm{ex}}$ represent the energies of uncoupled dipole, quadrupole and exciton resonances, $\gamma_{\mathrm{D}}, \gamma_{\mathrm{Q}}$ and $\gamma_{\mathrm{ex}}$ denote the dissipation rates of these modes, $g_{\mathrm{D}-\mathrm{Q}}$, $g_{\mathrm{D}-\mathrm{ex}}$ and $g_{\mathrm{Q}-\mathrm{ex}}$ stand for the dipole-quadrupole, dipole-exciton and quadrupole-exciton coupling strengths, respectively. 
In Figure $5 c$, the three mixed states, which are denoted as $E_{\mathrm{L}}, E_{\mathrm{M}}$ and $E_{\mathrm{H}}$ bands, are represented by the solid curves in the two-dimensional scattering spectra. The dotted lines represent the energies of the three uncoupled modes. It is noticed that the energies of the exciton mode $\left(E_{\mathrm{ex}}=2.016 \mathrm{eV}\right)$ and the quadrupole mode $\left(E_{\mathrm{Q}}=1.922 \mathrm{eV}\right)$ remain nearly unchanged with increasing $L_{\mathrm{s}}$. In Figure $5 d$, we show the simulation results and theoretical fittings for the dipole-quadrupole-exciton coupling. Furthermore, we present the Hopfield coefficients for dipole, quadrupole, exciton contributions to the three mixed state branches of the hybrid system in Figure 5e. For the coupling of three modes, the energy splitting depends not only on the coupling strengths of dipole-quadrupole, dipoleexciton and exciton-quadrupole but also on the energies and linewidths of these modes. Therefore, the analytical expressions for the eigenenergies of the polariton modes are not available. Therefore, neither is the criterion for strong coupling. We can extract the coupling strengths between two modes to be $g_{\mathrm{D}-\mathrm{Q}} \approx 124 \mathrm{meV}, g_{\mathrm{D}-\mathrm{ex}} \approx 64 \mathrm{meV}$, and $g_{\mathrm{Q}-\mathrm{ex}} \approx 32 \mathrm{meV}$ based on fitting. In addition, the minimum energy splitting between the $E_{\mathrm{L}}$ and $E_{\mathrm{H}}$ bands is derived to be $\Omega_{\mathrm{H}-\mathrm{L}} \approx 299 \mathrm{meV}$. This value is much larger than that observed in the coupling between a single GNR and a $W_{2}$ monolayer (see Figure 4), implying that the introduction of the dark quadrupole mode can really boost the plasmonexciton coupling. Apparently, a simplified strong coupling criterion is fulfilled in this case because $\Omega_{\mathrm{H}-\mathrm{L}}>\left(\gamma_{\mathrm{D}}+\gamma_{\mathrm{Q}}+\gamma_{\mathrm{ex}}\right) / 2 \approx 146.5 \mathrm{meV}$.

In order to confirm the strong coupling among dipole, quadrupole and exciton realized in a T-shaped GNR dimer placed on a $\mathrm{WS}_{2}$ monolayer, we need to examine not only the two-dimensional scattering spectrum but also the absorption spectrum of the T-shaped GNR dimer, as shown in Figure 6a. Apart from the three branches of hybrid states, one can see a narrow absorption band at $\sim 615 \mathrm{~nm}$. However, this absorption band disappears in the two-dimensional absorption spectrum of the corresponding T-shaped GNR dimer in the absence of the $\mathrm{WS}_{2}$ monolayer, which is shown in Figure 6b. This phenomenon indicates that the narrow absorption band may originate from the absorption of the excitons in the $\mathrm{WS}_{2}$ monolayer. This assignment is verified by inspecting the two-dimensional absorption spectrum obtained by subtracting the spectrum shown in Figure $6 \mathrm{~b}$ from that shown in Figure 6a, as shown in Figure 6c.

(a)

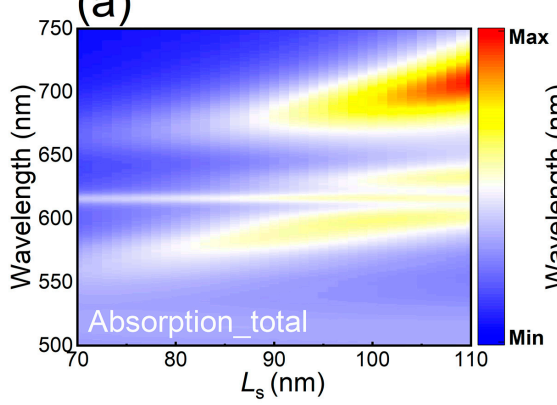

(b)

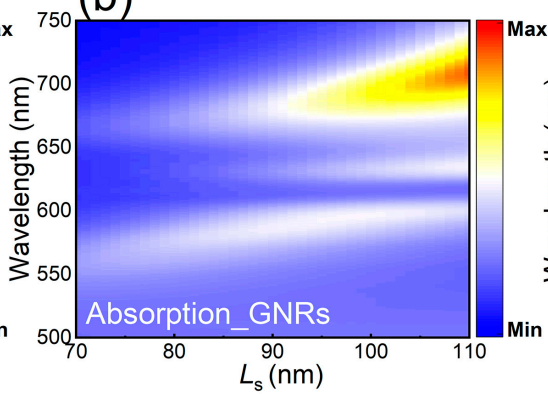

(c)

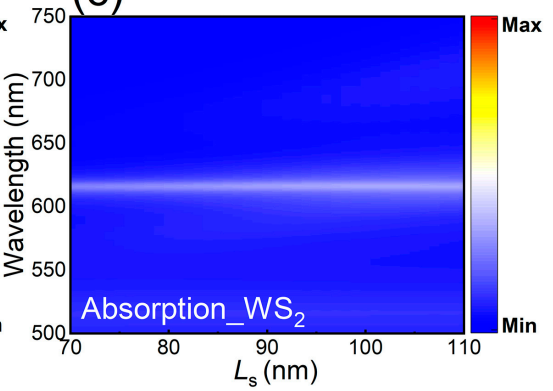

Figure 6. (a) Two-dimensional absorption spectrum calculated for a T-shaped GNR dimer with variant length of the short GNR placed on a $\mathrm{WS}_{2}$ monolayer. (b) Two-dimensional absorption spectrum calculated for a T-shaped GNR dimer with variant length of the short GNR. (c) Two-dimensional absorption spectrum obtained by subtracting the spectrum shown in (b) from that shown in (a).

Next, we examined the near-fields of the three mixed states and the electric field enhancements in all polaritons modes, as shown in Figure 7. Owing to the existence of the $\mathrm{WS}_{2}$ monolayer, the localization of electric field occurs on the contact surface. Therefore, we can identify the dipole mode excited in the short GNR and the quadrupole excited in the long GNR. It indicates that the dark quadrupole mode is indeed excited in this system, leading to the strong coupling among the dipole, quadrupole and exciton modes. If we inspected the electric field distributions at the scattering valley of $\lambda=615 \mathrm{~nm}$, we found that the electric field is mainly localized on the contact surface between the short GNR 
and the $\mathrm{WS}_{2}$ monolayer, quite similar to the coupling between a single GNR and a $\mathrm{WS}_{2}$ monolayer (see Figure 4). In contrast, the electric field enhancement on the contact surface between the long GNR and the $\mathrm{WS}_{2}$ monolayer become comparable at the scattering peaks of $\lambda=583$ and $678 \mathrm{~nm}$. In these cases, the electric field is mainly confined in the gap region between the two GNRs and the electric field enhancement between the long GNR and the monolayer $\mathrm{WS}_{2}$ is small. It means that the mode coupling in this system is dominated by dipole-exciton and dipole-quadrupole coupling, in good agreement with the coupling strengths derived from the coupled oscillator model (i.e., Equation (3)).

(a)
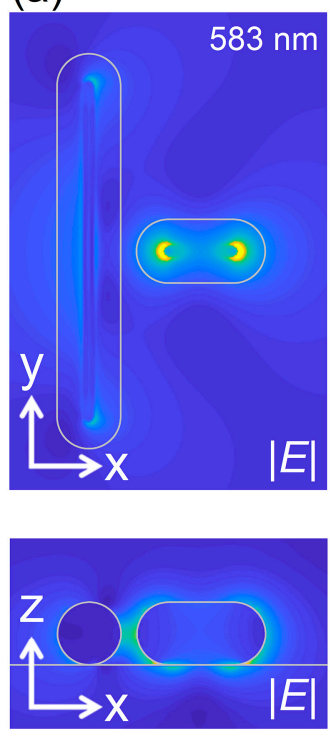

(b)
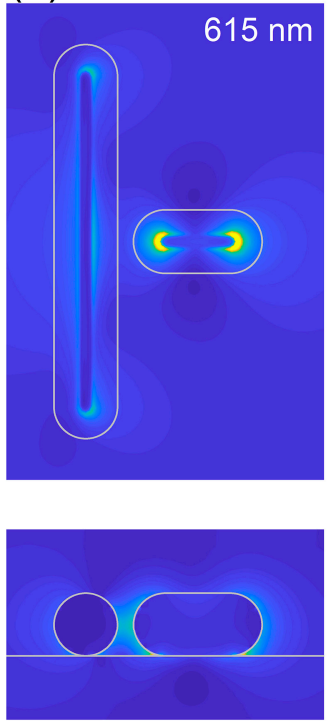

(c)
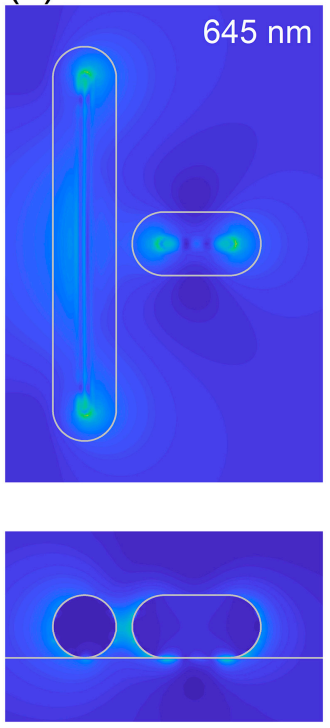

(d)

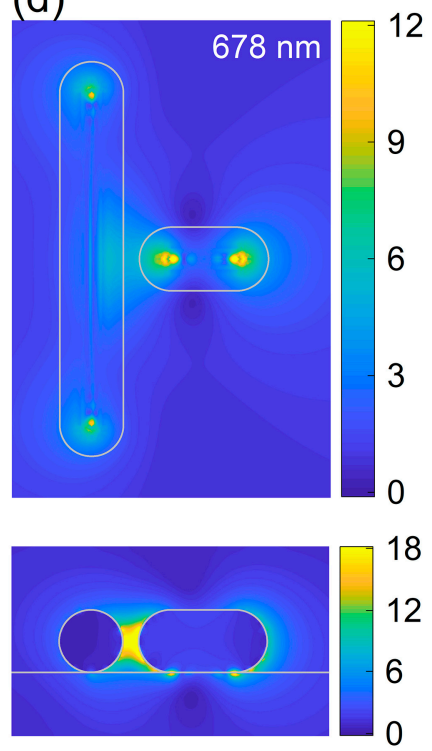

Figure 7. Electric field distributions in the XY (upper panel) and XZ planes (lower panel) calculated for the T-shaped GNR dimer placed on a $\mathrm{WS}_{2}$ monolayer with $L_{1}=248 \mathrm{~nm}, L_{\mathrm{s}}=81 \mathrm{~nm}$ and $w=10 \mathrm{~nm}$ at different wavelengths. (a) $\lambda=583 \mathrm{~nm}$, (b) $\lambda=615 \mathrm{~nm}$, (c) $\lambda=645 \mathrm{~nm}$ and (d) $\lambda=678 \mathrm{~nm}$.

To verify the feasibility of the proposed system on practical experiments, we calculated the scattering spectrum of the GNR dimer placed on a $\mathrm{SiO}_{2}$ substrate, as shown in Figure 8 . In this case, the $\mathrm{SiO}_{2}$ substrate was modeled as a semi-infinite layer with a refractive index of 1.5. In Figure 8, one can identify three scattering peaks in the scattering spectrum, implying that strong dipole-quadrupole-exciton coupling can also be realized in the presence of a $\mathrm{SiO}_{2}$ substrate. In addition, it is remarkable that the energy splitting between the low- and high-energy peaks appears to be larger in the presence of the $\mathrm{SiO}_{2}$. In indicates that strong dipole-quadrupole-exciton coupling achieved in a T-shaped GNR coupled with a $\mathrm{WS}_{2}$ monolayer proposed in this work can be implemented in experiments.

\section{Conclusions}

In summary, we proposed a novel strategy to enhance the plasmon-exciton coupling between the plasmons excited in GNRs and the excitons in two-dimensional materials. A T-shaped GNR dimer, which is composed of a long GNR and a short GNR arranged perpendicularly, was employed to achieve this goal through the excitation of the dark quadrupole mode in the long GNR. Three mixed states, which originate from the dipolequadruple-exciton coupling, are revealed in both the scattering and the absorption spectra of the GNR dimer placed on a $\mathrm{WS}_{2}$ monolayer. Rabi splitting as large as $299 \mathrm{meV}$ is observed, implying the strong coupling among the dipole, quadrupole and exciton modes. Our findings open new horizons for enhancing light-matter interaction and pave the wave for constructing novel nanoscale devices for practical applications. 


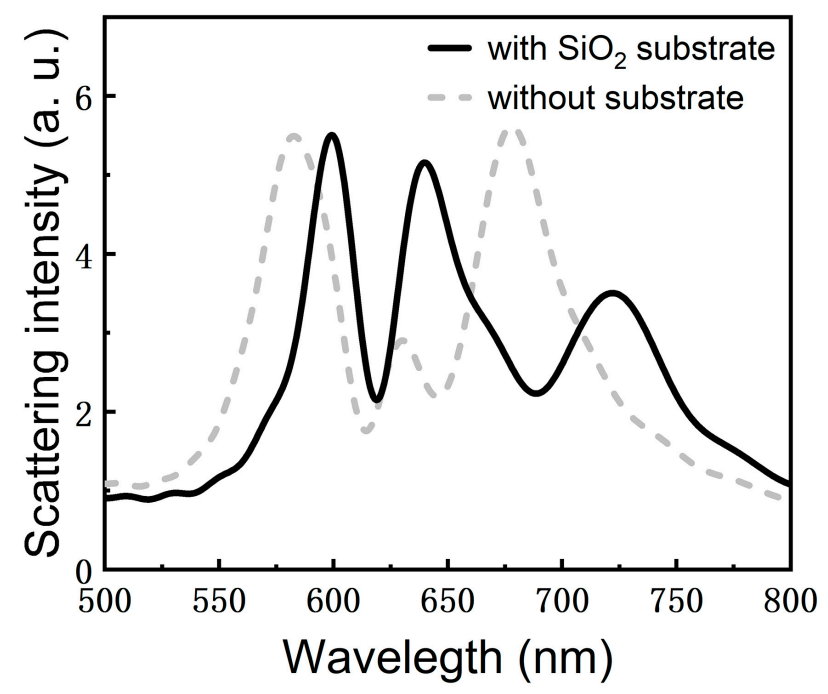

Figure 8. Scattering spectrum calculated for a GNR dimer placed on a $\mathrm{SiO}_{2}$ substrate with an embedded $\mathrm{WS}_{2}$ monolayer (black solid curve). The scattering spectrum of the hybrid structure in the absence of the substrate is also provided for comparison (dashed curve).

Author Contributions: Conceptualization, S.L. and F.D.; methodology, F.D.; software, H.P. and H.H.; investigation, H.P. and H.H.; validation, L.Z. and Y.M.; formal analysis, H.P., H.H., F.D. and S.L.; writing - original draft preparation, H.P. and H.H.; writing-review and editing, S.L. and F.D.; funding acquisition, S.L. All authors have read and agreed to the published version of the manuscript.

Funding: This work was financially supported by the National Natural Science Foundation of China (Grant Nos. 11674110 and 11874020) and the Natural Science Foundation of Guangdong Province, China (Grant Nos. 2016A030308010).

Data Availability Statement: The data presented in this study are available on request from the corresponding author.

Conflicts of Interest: The authors declare no conflict of interest.

\section{References}

1. Raimond, J.M.; Brune, M.; Haroche, S. Colloquium: Manipulating quantum entanglement with atoms and photons in a cavity. Rev. Mod. Phys. 2001, 73, 565-582. [CrossRef]

2. Mabuchi, H.; Doherty, A.C. Cavity quantum electrodynamics: Coherence in context. Science 2002, 298, 1372-1377. [CrossRef] [PubMed]

3. Kasprzak, J.; Richard, M.; Kundermann, S.; Baas, A.; Jeambrun, P.; Keeling, J.M.; Marchetti, F.M.; Szymanska, M.H.; Andre, R.; Staehli, J.L.; et al. Bose-Einstein condensation of exciton polaritons. Nature 2006, 443, 409-414. [CrossRef] [PubMed]

4. Torma, P.; Barnes, W.L. Strong coupling between surface plasmon polaritons and emitters: A review. Rep. Prog. Phys. 2015, 78, 013901. [CrossRef]

5. Sillanpaa, M.A.; Park, J.I.; Simmonds, R.W. Coherent quantum state storage and transfer between two phase qubits via a resonant cavity. Nature 2007, 449, 438-442. [CrossRef] [PubMed]

6. Chen, W.; Beck, K.M.; Bucker, R.; Gullans, M.; Lukin, M.D.; Tanji-Suzuki, H.; Vuletic, V. All-optical switch and transistor gated by one stored photon. Science 2013, 341, 768-770. [CrossRef] [PubMed]

7. Kena-Cohen, S.; Forrest, S.R. Room-temperature polariton lasing in an organic single-crystal microcavity. Nat. Photonics 2010, 4 , 371-375. [CrossRef]

8. Khitrova, G.; Gibbs, H.M.; Kira, M.; Koch, S.W.; Scherer, A. Vacuum Rabi splitting in semiconductors. Nat. Phys. 2006, 2, 81-90. [CrossRef]

9. Smolka, S.; Wuester, W.; Haupt, F.; Faelt, S.; Wegscheider, W.; Imamoglu, A. Cavity quantum electrodynamics with many-body states of a two-dimensional electron gas. Science 2014, 346, 332-335. [CrossRef]

10. Baranov, D.G.; Wersäll, M.; Cuadra, J.; Antosiewicz, T.J.; Shegai, T. Novel Nanostructures and Materials for Strong Light-Matter Interactions. ACS Photonics 2017, 5, 24-42. [CrossRef]

11. Purcell, E.M.; Torrey, H.C.; Pound, R.V. Resonance Absorption by Nuclear Magnetic Moments in a Solid. Phys. Rev. 1946, 69, 37-38. [CrossRef] 
12. Liu, M.; Lee, T.W.; Gray, S.K.; Guyot-Sionnest, P.; Pelton, M. Excitation of dark plasmons in metal nanoparticles by a localized emitter. Phys. Rev. Lett. 2009, 102, 107401. [CrossRef]

13. Sancho-Parramon, J.; Bosch, S. Dark modes and Fano resonances in plasmonic clusters excited by cylindrical vector beams. ACS Nano 2012, 6, 8415-8423. [CrossRef] [PubMed]

14. Huang, J.S.; Kern, J.; Geisler, P.; Weinmann, P.; Kamp, M.; Forchel, A.; Biagioni, P.; Hecht, B. Mode imaging and selection in strongly coupled nanoantennas. Nano Lett. 2010, 10, 2105-2110. [CrossRef] [PubMed]

15. Zhou, W.; Odom, T.W. Tunable subradiant lattice plasmons by out-of-plane dipolar interactions. Nat. Nanotechnol. 2011, 6, 423-427. [CrossRef] [PubMed]

16. Gao, Y.X.; Zhou, N.; Shi, Z.X.; Guo, X.; Tong, L.M. Dark dimer mode excitation and strong coupling with a nanorod dipole. Photonics Res. 2018, 6, 887-892. [CrossRef]

17. Du, K.; Li, P.; Gao, K.; Wang, H.; Yang, Z.; Zhang, W.; Xiao, F.; Chua, S.J.; Mei, T. Strong Coupling between Dark Plasmon and Anapole Modes. J. Phys. Chem. Lett. 2019, 10, 4699-4705. [CrossRef]

18. Ramasubramaniam, A. Large excitonic effects in monolayers of molybdenum and tungsten dichalcogenides. Phys. Rev. B 2012, 86, 115409. [CrossRef]

19. Ye, Z.; Cao, T.; O’Brien, K.; Zhu, H.; Yin, X.; Wang, Y.; Louie, S.G.; Zhang, X. Probing excitonic dark states in single-layer tungsten disulphide. Nature 2014, 513, 214-218. [CrossRef]

20. Li, Y.L.; Chernikov, A.; Zhang, X.; Rigosi, A.; Hill, H.M.; van der Zande, A.M.; Chenet, D.A.; Shih, E.M.; Hone, J.; Heinz, T.F. Measurement of the optical dielectric function of monolayer transition-metal dichalcogenides: $\mathrm{MoS}_{2}, \mathrm{MoSe}_{2}, \mathrm{WS}_{2}$, and WSe 2 Phys. Rev. B 2014, 90, 205422. [CrossRef]

21. Amani, M.; Taheri, P.; Addou, R.; Ahn, G.H.; Kiriya, D.; Lien, D.H.; Ager, J.W., 3rd; Wallace, R.M.; Javey, A. Recombination Kinetics and Effects of Superacid Treatment in Sulfur- and Selenium-Based Transition Metal Dichalcogenides. Nano Lett. 2016, 16, 2786-2791. [CrossRef]

22. Huang, L.; Li, G.; Gurarslan, A.; Yu, Y.; Kirste, R.; Guo, W.; Zhao, J.; Collazo, R.; Sitar, Z.; Parsons, G.N.; et al. Atomically Thin $\mathrm{MoS}_{2}$ Narrowband and Broadband Light Superabsorbers. ACS Nano 2016, 10, 7493-7499. [CrossRef]

23. Chernikov, A.; Berkelbach, T.C.; Hill, H.M.; Rigosi, A.; Li, Y.; Aslan, O.B.; Reichman, D.R.; Hybertsen, M.S.; Heinz, T.F. Exciton binding energy and nonhydrogenic Rydberg series in monolayer $\mathrm{WS}_{2}$. Phys. Rev. Lett. 2014, 113, 076802. [CrossRef]

24. Liu, X.; Galfsky, T.; Sun, Z.; Xia, F.; Lin, E.-C.; Lee, Y.-H.; Kéna-Cohen, S.; Menon, V.M. Strong light-matter coupling in two-dimensional atomic crystals. Nat. Photonics 2014, 9, 30-34. [CrossRef]

25. Bisht, A.; Cuadra, J.; Wersall, M.; Canales, A.; Antosiewicz, T.J.; Shegai, T. Collective Strong Light-Matter Coupling in Hierarchical Microcavity-Plasmon-Exciton Systems. Nano Lett. 2019, 19, 189-196. [CrossRef]

26. Lee, B.; Liu, W.; Naylor, C.H.; Park, J.; Malek, S.C.; Berger, J.S.; Johnson, A.T.C.; Agarwal, R. Electrical Tuning of ExcitonPlasmon Polariton Coupling in Monolayer $\mathrm{MoS}_{2}$ Integrated with Plasmonic Nanoantenna Lattice. Nano Lett. 2017, 17, 4541-4547. [CrossRef]

27. Qin, M.; Xiao, S.; Liu, W.; Ouyang, M.; Yu, T.; Wang, T.; Liao, Q. Strong coupling between excitons and magnetic dipole quasi-bound states in the continuum in $\mathrm{WS}_{2}-\mathrm{TiO}_{2}$ hybrid metasurfaces. Opt. Express 2021, 29, 18026-18036. [CrossRef]

28. Wen, J.; Wang, H.; Wang, W.; Deng, Z.; Zhuang, C.; Zhang, Y.; Liu, F.; She, J.; Chen, J.; Chen, H.; et al. Room-Temperature Strong Light-Matter Interaction with Active Control in Single Plasmonic Nanorod Coupled with Two-Dimensional Atomic Crystals. Nano Lett. 2017, 17, 4689-4697. [CrossRef]

29. Geisler, M.; Cui, X.M.; Wang, J.F.; Rindzevicius, T.; Gammelgaard, L.; Jessen, B.S.; Goncalves, P.A.D.; Todisco, F.; Boggild, P.; Boisen, A.; et al. Single-Crystalline Gold Nanodisks on $\mathrm{WS}_{2}$ Mono- and Multilayers for Strong Coupling at Room Temperature. ACS Photonics 2019, 6, 994-1001. [CrossRef]

30. Cuadra, J.; Baranov, D.G.; Wersall, M.; Verre, R.; Antosiewicz, T.J.; Shegai, T. Observation of Tunable Charged Exciton Polaritons in Hybrid Monolayer $W_{2}$-Plasmonic Nanoantenna System. Nano Lett. 2018, 18, 1777-1785. [CrossRef]

31. Munkhbat, B.; Baranov, D.G.; Bisht, A.; Hoque, M.A.; Karpiak, B.; Dash, S.P.; Shegai, T. Electrical Control of Hybrid Monolayer Tungsten Disulfide-Plasmonic Nanoantenna Light-Matter States at Cryogenic and Room Temperatures. ACS Nano 2020, 14, 1196-1206. [CrossRef]

32. Zheng, D.; Zhang, S.; Deng, Q.; Kang, M.; Nordlander, P.; Xu, H. Manipulating Coherent Plasmon-Exciton Interaction in a Single Silver Nanorod on Monolayer WSe 2 . Nano Lett. 2017, 17, 3809-3814. [CrossRef]

33. Lepeshov, S.; Wang, M.; Krasnok, A.; Kotov, O.; Zhang, T.; Liu, H.; Jiang, T.; Korgel, B.; Terrones, M.; Zheng, Y.; et al. Tunable Resonance Coupling in Single Si Nanoparticle-Monolayer $W_{2}$ Structures. ACS Appl. Mater. Interfaces 2018, 10, 16690-16697. [CrossRef]

34. Yan, X.; Wei, H. Strong plasmon-exciton coupling between lithographically defined single metal nanoparticles and monolayer $\mathrm{WSe}_{2}$. Nanoscale 2020, 12, 9708-9716. [CrossRef]

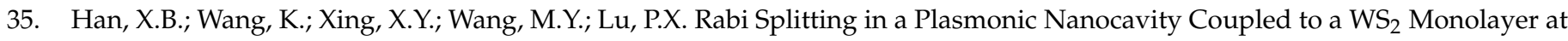
Room Temperature. ACS Photonics 2018, 5, 3970-3976. [CrossRef]

36. Kleemann, M.E.; Chikkaraddy, R.; Alexeev, E.M.; Kos, D.; Carnegie, C.; Deacon, W.; de Pury, A.C.; Grosse, C.; de Nijs, B.; Mertens, J.; et al. Strong-coupling of $\mathrm{WSe}_{2}$ in ultra-compact plasmonic nanocavities at room temperature. Nat. Commun. 2017, 8 , 1296. [CrossRef] [PubMed] 
37. Hou, S.; Tobing, L.Y.M.; Wang, X.; Xie, Z.; Yu, J.; Zhou, J.; Zhang, D.; Dang, C.; Coquet, P.; Tay, B.K.; et al. Manipulating Coherent Light-Matter Interaction: Continuous Transition between Strong Coupling and Weak Coupling in MoS $_{2}$ Monolayer Coupled with Plasmonic Nanocavities. Adv. Opt. Mater. 2019, 7, 1900857. [CrossRef]

38. Huang, H.X.; Deng, F.; Xiang, J.; Li, S.L.; Lan, S. Plasmon-exciton coupling in dielectric-metal hybrid nanocavities with an embedded two-dimensional material. Appl. Surf. Sci. 2021, 542, 148660. [CrossRef]

39. Deng, F.; Liu, H.; Xu, L.; Lan, S.; Miroshnichenko, A.E. Strong Exciton-Plasmon Coupling in a WS 2 Monolayer on Au Film Hybrid Structures Mediated by Liquid Ga Nanoparticles. Laser Photonics Rev. 2020, 14, 1900420. [CrossRef]

40. Xie, P.; Li, D.M.; Chen, Y.X.; Chang, P.J.; Zhang, H.; Yi, J.M.; Wang, W. Enhanced coherent interaction between monolayer WS 2 and film-coupled nanocube open cavity with suppressed incoherent damping pathway. Phys. Rev. B 2020, 102, 115430. [CrossRef]

41. Verre, R.; Baranov, D.G.; Munkhbat, B.; Cuadra, J.; Kall, M.; Shegai, T. Transition metal dichalcogenide nanodisks as high-index dielectric Mie nanoresonators. Nat. Nanotechnol. 2019, 14, 679-683. [CrossRef] [PubMed]

42. Rousseaux, B.; Baranov, D.G.; Antosiewicz, T.J.; Shegai, T.; Johansson, G. Strong coupling as an interplay of quantum emitter hybridization with plasmonic dark and bright modes. Phys. Rev. Res. 2020, 2, 033056. [CrossRef]

43. Li, B.W.; Zu, S.; Zhang, Z.P.; Zheng, L.H.; Jiang, Q.; Du, B.W.; Luo, Y.; Gong, Y.J.; Zhang, Y.F.; Lin, F.; et al. Large Rabi splitting obtained in $\mathrm{Ag}-\mathrm{WS}_{2}$ strong-coupling heterostructure with optical microcavity at room temperature. Opto Electron. Adv. 2019, 2, 19000801-19000809. [CrossRef]

44. Zhang, H.; Abhiraman, B.; Zhang, Q.; Miao, J.; Jo, K.; Roccasecca, S.; Knight, M.W.; Davoyan, A.R.; Jariwala, D. Hybrid exciton-plasmon-polaritons in van der Waals semiconductor gratings. Nat. Commun. 2020, 11, 3552. [CrossRef]

45. As'ham, K.; Al-Ani, I.; Huang, L.; Miroshnichenko, A.E.; Hattori, H.T. Boosting Strong Coupling in a Hybrid WSe 2 MonolayerAnapole-Plasmon System. ACS Photonics 2021, 8, 489-496. [CrossRef]

46. Johnson, P.B.; Christy, R.W. Optical Constants of the Noble Metals. Phys. Rev. B 1972, 6, 4370-4379. [CrossRef]

47. Zhang, S.; Chen, L.; Huang, Y.; Xu, H. Reduced linewidth multipolar plasmon resonances in metal nanorods and related applications. Nanoscale 2013, 5, 6985-6991. [CrossRef] [PubMed]

48. Huang, L.; Krasnok, A.; Alu, A.; Yu, Y.; Neshev, D.; Miroshnichenko, A.E. Enhanced Light-Matter Interaction in Two-Dimensional Transition Metal Dichalcogenides. arXiv 2021, arXiv:2103.11064.

49. Hopfield, J.J. Theory of the Contribution of Excitons to the Complex Dielectric Constant of Crystals. Phys. Rev. 1958, 112, 1555-1567. [CrossRef]

50. Zengin, G.; Wersall, M.; Nilsson, S.; Antosiewicz, T.J.; Kall, M.; Shegai, T. Realizing Strong Light-Matter Interactions between Single-Nanoparticle Plasmons and Molecular Excitons at Ambient Conditions. Phys. Rev. Lett. 2015, 114, 157401. [CrossRef] [PubMed]

51. Savasta, S.; Saija, R.; Ridolfo, A.; Di Stefano, O.; Denti, P.; Borghese, F. Nanopolaritons: Vacuum Rabi splitting with a single quantum dot in the center of a dimer nanoantenna. ACS Nano 2010, 4, 6369-6376. [CrossRef] [PubMed] 